\title{
Hanging Bladder calculi Secondary to Misplaced Surgical Suture
}

\author{
Ali Mahdavi ${ }^{1,} ;$ Hasan Mostafavi ${ }^{2}$ \\ ${ }_{1}^{1}$ Department of Radiology, Tehran University of Medical Sciences, Tehran, Iran \\ ${ }^{2}$ Department of Radiology, Iran University of Medical Sciences, Tehran, IR Iran \\ *Corresponding author: Ali Mahdavi, Department of Radiology, Tehran University of Medical Sciences, Tehran, Iran. Tel: +98-9121508905, E-mail: mahdavioali@yahoo.com
}

Received: March 25, 2013; Revised: May 21, 2013; Accepted: June 3, 2013

\begin{abstract}
Bladder calculi, a rare condition in the pediatric population, occur most commonly as a result of either migration from the kidney or urinary stasis in the bladder. We report the case of a3-year-old boy with recurrent urinary tract infections (UTI) secondary to bladder calculi formation on the sutures from a previous herniorrhaphy.A3-year-old boy with previous history of herniorrhaphy presented with recurrent episodes of urinary tract infection, resistant to antibiotic therapy. Physical examination was unremarkable. Ultrasonography (US) showed an echogenic fixed intra-luminal lesion in the bladder. Cystoscopic evaluation was performed and confirmed presence of calculi forming around several permanent silk sutures fixed to the bladder wall. The patient undergone cystotomy and the calculi were resected. The stone analysis revealed $80 \%$ uric acid calculi. The final diagnosis was of bladder calculi due to remnant suture from past herniorrhaphy.
\end{abstract}

Keywords:Urinary Bladder Calculi; Urinary Tract Infections; Sutures; Herniorrhaphy

\section{Introduction}

Bladder calculi, a rare condition in the pediatric population, occur most commonly as a result of either migration from the kidney or urinary stasis in the bladder. Previous pelvic or inguinal surgery with non-resorbable sutures, especially in the proximity of the bladder, may complicate with lithiasis, a starting point for recurrent UTI. Here, we report a 3-year-old boy with previous history of herniorrhaphy presented with recurrent episodes of urinary tract infection, resistant to antibiotic therapy. Physical examination was unremarkable. Ultrasonography (US) showed an echogenic fixed intra-luminal lesion in the bladder. Cystoscopic evaluation was performed and confirmed presence of calculi forming around several permanent silk sutures fixed to the bladder wall.

\section{Case Presentation}

A 3-year-old boy was referred to our hospital for evaluation of recurrent urinary tract infection (UTI) since about two and half years prior to admission. The patient had undergone herniorrhaphy procedure when he was 5 months old and he experienced the first episode of UTI just 1 month after the surgery. After that, he developed four additional episodes of UTI, requiring hospitalization and antibiotic therapy. Three months before admission in our center, he suffered from persistent UTI with Pseudomonas aeruginosa ( $P$. aeruginosa), resistant to antibiotic therapy. Upon admission, the patient was a well-developed 3-year-old boy, with normal developmental milestones, without urinary symptoms. He had several periods of fever and chills, without voiding dys- function. The physical examination was unremarkable. Dimercaptosuccinic acid (DMSA) scan showed no evidence of renal parenchymal scarring. The patient had a positive urine culture for $P$. aeruginosa. The serum blood urea nitrogen (BUN) and creatinine levels were normal. The intravenous urography (IVU) only revealed duplication of the right pyelocaliceal system. In the ultrasound (US) examination, multiple confluent linear echogenicities (10-20mm) with posterior acoustic shadows, located inside the bladder and fixed to the antero-superior wall during changes in patient position, were observed (Figure $1 \mathrm{~A}$ ). On color doppler US, a small vascular flow was observed in the posterior acoustic shadow, making it difficult to make the distinction between bladder tumor or stone aggregate with ring down artifact (Figure $1 \mathrm{~B})$. Based on ultrasound results, cystoscopy was performed to uncover the underlying cause, and the presence of multiple fixed calculi on the bladder dome was confirmed. Transurethral resection of the calculi was not possible and the patient underwent open cystostomy, which revealed multiple bladder calculi forming around several permanent silk sutures fixed to the bladder wall (from the previous herniorrhaphy). These sutures had been aberrantly included in the bladder wall during the remote right herniorrhaphy, as a starting point for bladder stone formation (Figure 2). The bladder calculi were resected and analysis revealed $80 \%$ uric acid composition. After surgery, the UTI resolved and the patient discharged from hospital with good condition and negative urine culture.

Copyright (C) 2015, Tehran University of Medical Sciences and Iranian Society of Radiology. This is an open-access article distributed under the terms of the Creative Commons Attribution-NonCommercial 4.0 International License (http://creativecommons.org/licenses/by-nc/4.0/) which permits copy and redistribute the material just in noncommercial usages, provided the original work is properly cited. 

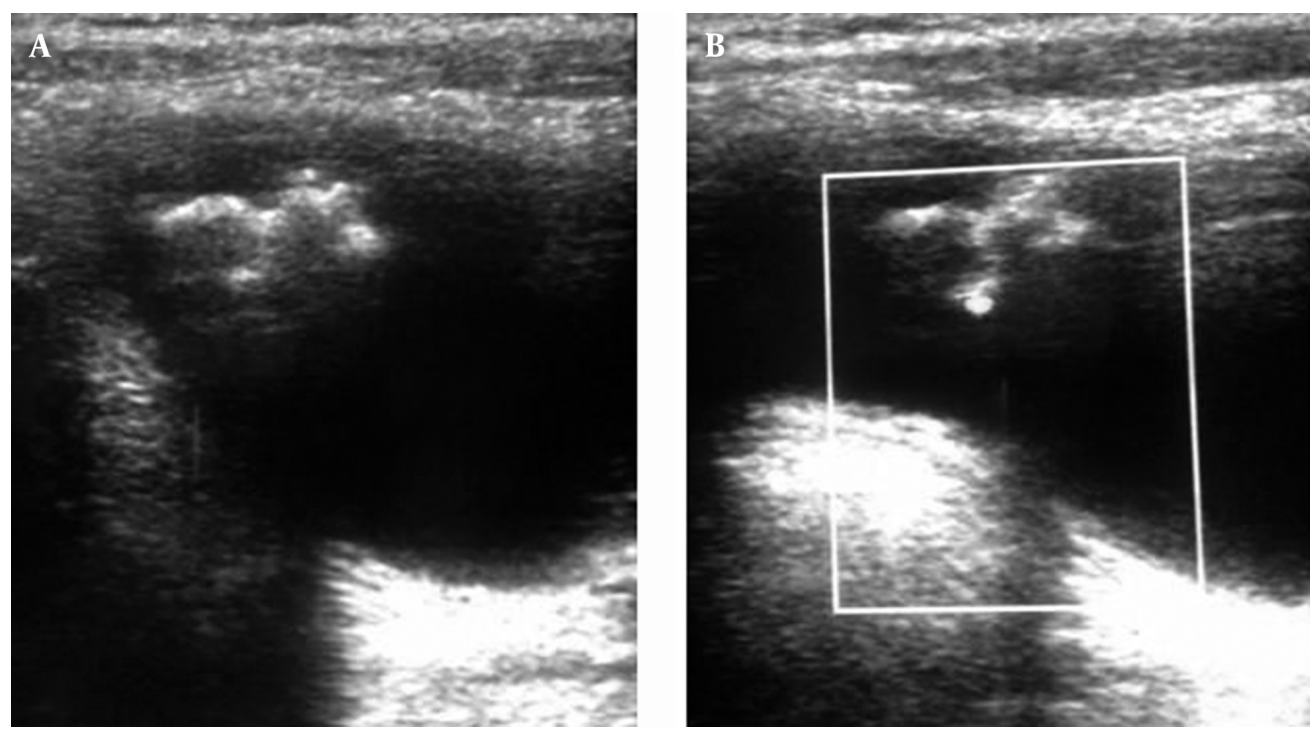

Figure 1. A 3-year-old boy with recurrent UTI and history of herniorrhaphy. A,B, Ultrasound exam revealed echogenic foci within bladder suspicious of calculi
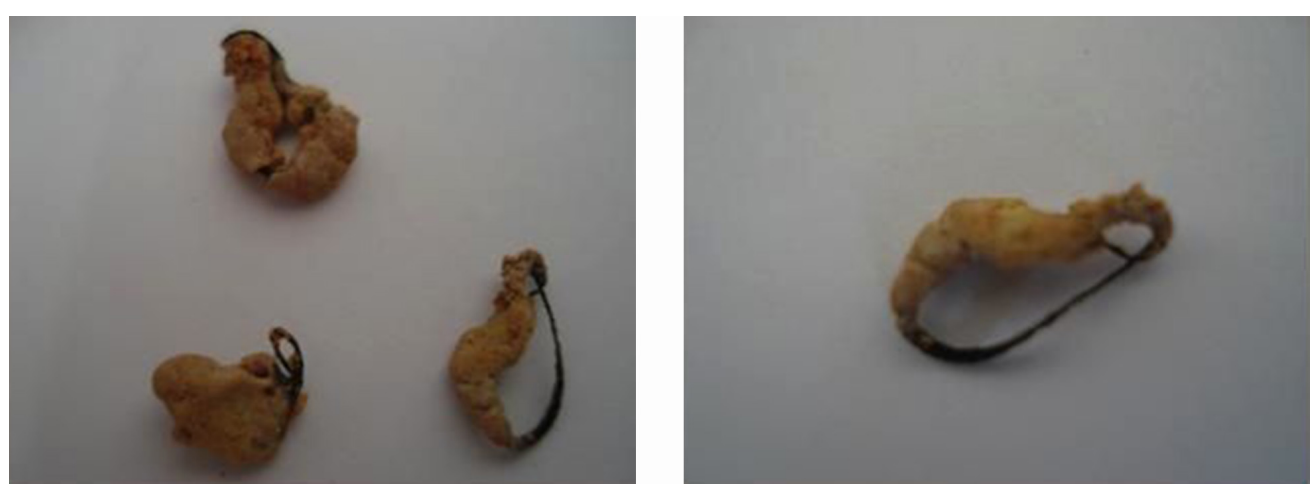

Figure 2. Urinary calculi formed around surgical sutures remnant within bladder wall

\section{Discussion}

Bladder stone is rare in the pediatric population. Bladder calculi occur most commonly as a result of either migration from the kidney or urinary stasis in the bladder. Urinary stasis is usually related to bladder outlet obstruction, cystocele, neurogenic bladder, or a foreign body in the bladder (1). Urinary tract stones and urinary tract infection are strongly associated. Infection is implicated as the cause of stones in about $15 \%$ of stone formers, and the development of infection can complicate the management of preexistent calculi. Left untreated, both situations can result in loss of kidney function, and may become, although rarely, life threatening conditions (2). On US, a mobile, echogenic focus with distal acoustic shadowing will be seen in the bladder. If the stone is large, edema of the ureteral orifices and thickening of the bladder wall may be seen. Occasionally, stones can adhere to the bladder wall because of adjacent in- flammation, and these are known as "hanging" bladder stones (1). Foreign bodies, such as surgical sutures, may act as a leading point for developing bladder calculi. These stones are typically non-mobile and present as hanging fixed echogenicitie son US. On the other hand, herniorrhaphy is one of the most commonly performed operations worldwide. Iatrogenic bladder injury is a rare complication of this surgery. Incorrect deep suturing or surgical mesh used in inguinal herniorrhaphy may involve the bladder wall and lumen, and intraluminal portion can act as a nidus for stone formation $(3,4)$. Based on literature reviews, other surgical operations, such as extrophy repair (5), stress urinary incontinence surgery (6), prostatectomy (7), caesarian section (8) etc., may also cause bladder stone formation due to application of non-absorbable sutures, like silk sutures. Even though, most of these complications occur in adult population, 
the review of our patient's medical history revealed the etiology. As far as we know, it is the first pediatric case of iatrogenic bladder calculi forming around the surgical sutures remnants. Here, we reported a 3-year-old boy who suffered from recurrent UTI with unknown origin, beginning just after herniorrhaphy surgery. The underlying etiology was underdiagnosed for about two and half years. Finally, in our department, aberrantly positioned surgical sutures remnant into the bladder wall were discovered after cystostomy, as a nidus for stone formation and the cause of recurrent UTI. The differential diagnosis for multiple linear fixed echogenicities in the bladder, with posterior acoustic shadow, includes multiple bladder calculi, calcified bladder tumor, foreign bodies, bladder wall inflammation and infection. Each of these can cause a mass like lesion in the bladder. Foreign bodies in the bladder wall are extremely rare, and a careful review of the patient's medical history is necessary when foreign bodies are suspected. The patient underwent open surgical excision of the suture remnants and surrounding calculi. After that, antibiotic therapy with held and patient's UTI resolved. Ultrasound examination was performed and confirmed the total resection of bladder calculi without any evidence of bladder mucosal thickening. Urinary culture obtained few days later showed no bacterial growth and the patient discharged from hospital with good condition. Bladder stone analysis performed several days later reported uric-acid stones. From the clinical point of view, the physicians must always pay attention to find the etiology of recurrent UTI. Complete past medical history taking and physical examination remain the most precious tools, despite the new advances in diagnostic modalities. However, the presenting patient's problem remained undiagnosed for about two and half years, despite several recurrent UTI episodes. Fortunately, the underlying etiology was found during US and the patient recovered without any evident parenchymal scars. From a radiological point of view, most bladder stones are frequently mobile and seen in dependent portion of the bladder. For non-mobile fixed stones, such as the presented case, we must always be aware of other etiologies, such as foreign bodies or inflammatory processes (1). Initially, we considered that the multiple echogenicities with acoustic shadows were related to a calcified bladder tumor, because of the focal doppler signal in the lesion. Nevertheless, one must be aware of the possibility of hanging stones or foreign bodies, especially in patients with a positive history for previous surgery in the pelvi cor inguinal regions. Suture remnants in the bladder have an important role in recurrent cystolithiasis in animal studies (9). This case confirms the lithogenic nature of non-absorbable sutures in contact with urine, in the pediatric population. These stones are extremely prone to cause urinary infections and surgery is the mainstay of treatment for such infections. Stone removal is the goal and a range of minimally invasive treatments are available (2). However, to avoid serious complications, surgeons must be careful and to use absorbable sutures in deep structures and also to take more attention to avoid bladder injury during nearby operations.

\section{Acknowledgements}

Special thanks to Dr. Hassan Otoukesh for his support during this case report.

\section{References}

1. Thurston W, Wilson SR. Diagnostic Ultrasound. 3 th edUSA: Mosby: 2005.

2. Thomas B, Tolley D. Concurrent urinary tract infection and stone disease: pathogenesis, diagnosis and management. Nat Clin Pract Urol. 2008;5(12):668-75.

3. Colegate-Stone TJ, Raymond T, Khot U, Dickinson IK, Parker MC Laparoscopic management of iatrogenic bladder injury and bladder stone formation following laparoscopic inguinal herniorrhaphy. Hernia. 2008;12(4):429-30.

4. Ngo T. Surgical mesh used for an inguinal herniorrhaphy acting as a nidus for a bladder calculus. Int J Urol.2006;13(9):1249-50.

5. Rub R, Madeb R, Morgenstern S, Ben-Chaim J, Avidor Y. Development of a large bladder calculus on sutures used for pubic bone closure following extrophy repair. World J Urol. 2001;19(4):261-2.

6. Huang WC, Yang JM. Sonographic appearance of a bladder calculus secondary to a suture from a bladder neck suspension. $J$ Ultrasound Med. 2002;21(11):1303-5.

7. Cursio R, Choquenet C. Iatrogenic bladder stone formation on absorbable suture 3-years after radical prostatectomy. Minerva Urol Nefrol. 2002;54(2):127-8.

8. Su CM, Lin HY, Li CC, Chou YH, Huang CH. Bladder stone in a woman after cesarean section: a case report. Kaohsiung J Med Sci. 2003;19(1):42-4.

9. Kosan M, Gonulalan U, Ozturk B, Kulacoglu S, Erguder I, Akdemir O, et al. Tissue reactions of suture materials (polyglactine 910, chromed catgut and polydioxanone) on rat bladder wall and their role in bladder stone formation. Urol Res. 2008;36(1):43-9. 